\title{
Protocoles de prise en charge des incidents d'expositions internes au plutonium dans un service médical d'installation nucléaire de base : élaboration - mise en place - évaluation - validation de 1996 à 2002
}

\author{
N. BLANCHIN ${ }^{1}$, S. DESLOIRES ${ }^{1}$, L. GRAPPIN ${ }^{1}$, A.-M. GUILLERMIN ${ }^{1}$,

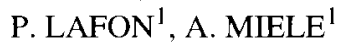

(Manuscrit reçu le 19 septembre 2003, accepté le 12 janvier 2004)

RÉSUMÉ Le service de santé au travail du CEA/CADARACHE est un service médical de site chargé de la surveillance radiologique de l'exposition interne de l'ensemble du personnel travaillant sur le centre de Cadarache. Dans notre activité spécifique de surveillance du risque radiologique prédomine la gestion des expositions aux actinides, plutonium et américium principalement, avec environ 600 agents concernés. Nous présentons les protocoles de prise en charge des incidents de contamination que nous avons élaborés et mis en place en 1996 ainsi que le retour d'expérience sur plus de 1500 évènements gérés jusqu'à fin 2002.

ABSTRACT Protocols in an occupational medical facility for the management of internal plutonium exposure incidents in a nuclear plant: development - application analysis - validation from 1996 to 2002.

The occupational health service of the CEA/CADARACHE is a medical unit attached to the company and is assigned to the monitoring of internal exposure of the whole staff at the plant. In our specific practice of the radiological risk survey, the management of actinide exposures which concerns about 600 workers is predominant. We present herein the protocols for the monitoring of internal exposure incidents that we elaborated and set up in 1996, and our experience on 1500 events managed up to the end of 2002.

\section{Introduction}

La gestion du risque radiologique par le médecin d'INB doit concilier les exigences médicales (prescription des examens nécessaires à l'évaluation dosimétrique, traitement, exclusion de poste) et leur acceptabilité par les salariés et l'employeur. L'importance du nombre d'évènements de contamination par transuraniens pris en charge par le service médical du centre de Cadarache

1 Service de santé au travail, CEA Cadarache, 13108 Saint-Paul-lez-Durance, France. 
(environ 200 par an) renforce cette exigence et nous a conduit à élaborer des protocoles de prise en charge adaptés bâtis sur une conduite médicale pertinente et cohérente.

Nous nous proposons de présenter ces protocoles et de les discuter avec le recul de 8 ans de mise en pratique et plus de 1500 dossiers d'incidents de contamination gérés par le service médical.

Sur la période considérée (1996-2002), la réglementation applicable repose sur le décret $n^{\circ} 66-450$ du 20 juin 1966 relatif aux principes généraux de protection contre les rayonnements ionisants. Les limites annuelles d'incorporation (LAI) définissent ( $c f$. Annexe IV) les limites réglementaires en matière d'exposition interne et permettent le calcul des limites dérivées de concentration atmosphérique (LDCA). Nous avons calculé une LAI-mélange à partir de la composition isotopique moyenne des produits manipulés sur nos installations. Les modèles d'estimation de l'incorporation sont ceux de la CIPR (ICRP, 1989) repris dans les rapports CEA de J. Piechowski et al. (1992) en se référant à la surveillance dite « spéciale ». Les paramètres physico-chimiques utilisés par défaut pour les calculs sont ceux de la publication 54 de la CIPR (ICRP, 1989).

L'application du décret $\mathrm{n}^{\circ}$ 2003-296 du 31 mars 2003 doit nous conduire à intégrer de nouvelles valeurs de référence à nos protocoles. Cette période transitoire d'évolution réglementaire a paru opportune pour faire le point sur les années précédentes et nous guider dans l'établissement de nos futures consignes.

\section{Le contexte}

À Cadarache, le plutonium est surtout utilisé dans l'établissement COGEMA qui produit pour les centrales nucléaires du combustible MOX (contenant 3 à $7 \%$ de plutonium). Le produit est essentiellement sous forme solide (oxyde), mais existe aussi en solution notamment nitrique. Il est confiné tout au long de la chaîne de fabrication. Les interventions sont soit automatisées, soit manuelles par l'intermédiaire de boites à gants (BAG). Un confinement dynamique est assuré par une ventilation de type nucléaire.

Les pertes de confinement (anomalie lors d'un transfert, ouverture accidentelle d'un colis, défectuosité d'un gant...) peuvent entrainer une dispersion atmosphérique ou de surface, source de contamination radiologique potentielle du personnel travaillant à proximité.

L'atmosphère de travail est surveillée en continu par des balises de détection de type EDGAR (Cérat, 1994) avec alarmes asservies. Par convention interne, 
le seuil d'alarme des balises est réglé pour le plutonium sur 5 limites dérivées de concentration atmosphérique (LDCA) en pic et 2 LDCA.h en intégrant le niveau de la montée atmosphérique sur le temps. 2000 LDCA.h correspondent à une LAI (2000 heures étant le nombre d'heures travaillées par an de référence).

Des appareils de détection surfacique sont placés en différents points selon une logique de radioprotection : contrôle des mains, des pieds, des vêtements aux différents changements de zone. Les locaux (sols, BAG...) sont par ailleurs périodiquement contrôlés par le Service de protection contre les rayonnements (SPR). Les radio-protectionnistes interviennent pour toute anomalie à caractère radiologique : sur un déclenchement d'alarme atmosphérique, sur un appel d'un opérateur ayant constaté une contamination lors d'un contrôle cutané ou vestimentaire, sur un contrôle de surface de travail positif, enfin pour toute plaie même minime en zone à risque de contamination.

Un «évènement radiologique » est classé en «incident radiologique » par le service de radioprotection lorsque le niveau atmosphérique atteint $5 \mathrm{LDCA} \cdot \mathrm{h}$. Les autres critères de classification sont les niveaux de contamination externe de la personne : supérieure à $5 \mathrm{~Bq}$ au niveau de la tête, $15 \mathrm{~Bq}$ au niveau du corps, $50 \mathrm{~Bq}$ sur les vêtements.

L'organisation des secours du Centre permet au Service médical et au Laboratoire d'analyses de biologie médicale (LABM) la prise en charge dans un délai très court des salariés contaminés.

\section{3. Élaboration et mise en place de protocoles de gestion des incidents de contamination}

\subsection{L'historique}

L'évolution de l'établissement COGEMA de Cadarache permet de mieux comprendre la genèse de nos protocoles :

- début des années 90 : une installation CEA de recherche sur le combustible est transférée à la COGEMA et transformée en unité de production ;

- passage en horaires postés $(3 \times 8$ avec 5 équipes) pour permettre la mise en place d'un procédé de fabrication en continu et l'augmentation progressive de production (40 tonnes produites annuellement à partir de 1998) ;

- augmentation des besoins en personnel (effectif total 350 salariés) et embauche d'une centaine de personnes dont la moitié environ issue des mines d'uranium qui cessent leur activité à cette époque ;

- enfin, surcroît d'activité, avec recours régulier à des salariés en contrat à durée déterminée. 
L'augmentation rapide de production, avec un pic en 1996 et 1997 et les évolutions techniques nécessaires ont été à l'origine d'un accroissement important du nombre de pertes de confinement. Parallèlement, le renouvellement important des salariés ayant une hétérogénéité de culture et de pratiques a limité la mise en place de formations au risque nucléaire par compagnonnage. Ce contexte s'est traduit par des demandes croissantes à la fois des salariés et de la hiérarchie, sur la gestion des incidents radiologiques avec une préoccupation forte sur la prise en compte du risque par le service médical.

Enfin, la mise en place des astreintes médicales pour la prise en charge des évènements radiologiques était récente et il n'y avait pas de protocole établi.

\section{2. Élaboration des protocoles}

Nous avons commencé à réfléchir sur nos pratiques en nous appuyant sur différents constats mis à jour lors de discussions entre hiérarchie COGEMA, salariés, Comité d'hygiène et sécurité et des conditions de travail (CHSCT), agents de radioprotection, infirmiers et médecins :

- les salariés victimes d'un incident radiologique, parfois la nuit, étaient inquiets et posaient des questions sur les examens, le traitement, les répercussions éventuelles sur leur santé et leur avenir professionnel. Cette inquiétude était reçue par l'infirmier de permanence peu préparé à y répondre ;

- l'employeur, gêné dans son activité de production par les décisions d'exclusion de leurs postes de travail des salariés impliqués dans un incident demandait à l'équipe médicale de les justifier et d'avoir une attitude homogène ;

- le recueil des excréta est contraignant pour les salariés ; des prescriptions trop fréquentes risquent d'être mal suivies ;

- les liaisons dans les suites immédiates de l'incident avec les agents de radioprotection n'étaient pas formalisées et cela pouvait retarder la prise en charge médicale.

L'analyse de cette situation et du retour d'expérience des incidents antérieurs, nous a orientés vers la décision de bâtir des conduites à tenir (protocoles) pour la gestion des incidents radiologiques en distinguant d'une part les expositions par inhalation et d'autre part les plaies.

\subsubsection{Expositions par inhalation}

Nous avons retenu deux paramètres d'orientation initiale, représentatifs et rapidement disponibles évaluant l'ordre de grandeur de l'évènement :

- le prélèvement de mucus nasal (PN) est un frottis d'exécution simple, le résultat obtenu par comptage direct alpha : est dit " positif» au-dessus d'un seuil d'action $(0,7 \mathrm{~Bq}$ pour notre laboratoire) et «négatif » au-dessous. 


\section{TABLEAU I}

Correspondance entre les mesures atmosphériques au poste de travail, la limite de détection des examens de laboratoire* et la LAI.

Correspondence between atmospheric measurements at workplace, detection limits of the laboratory examinations and the ALI.

$\begin{array}{lll}\begin{array}{l}\text { Mesures SPR } \\ \text { LDCA.h }\end{array} & \text { Mesures LABM } & \text { Limites réglementaires } \\ & \text { et niveaux opérationnels } \\ \text { en surveillance spéciale }\end{array}$

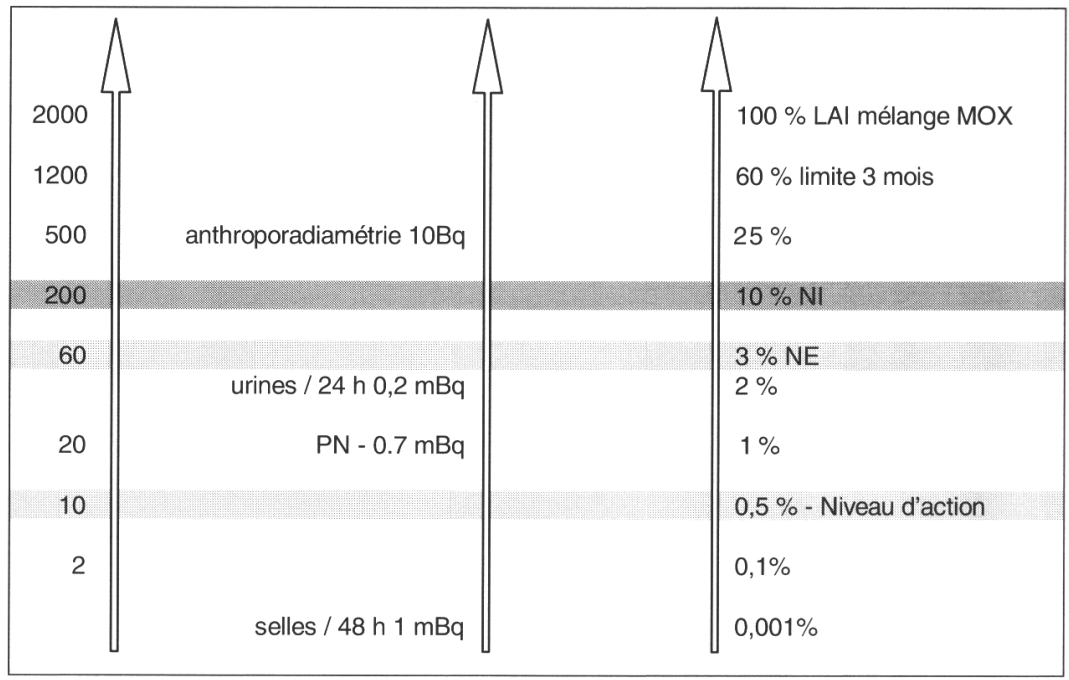

* L'interprétation des examens de laboratoire se fait selon les modèles de la publication 54 de la CIPR en surveillance spéciale pour la classe $Y$ dans le mode d'incorporation par inhalation.

Un PN positif est le témoin d'un dépôt du contaminant au niveau de la muqueuse nasale; il est le premier indicateur d'une incorporation ;

- le niveau de concentration atmosphérique pondéré par le temps de l'exposition exprimé en LDCA·h. Il s'agit d'un indicateur prédictif de l'exposition cumulée pendant la durée de l'évènement. La limite annuelle d'incorporation correspond à 2000 LDCA.h. La valeur donnée en LDCA.h peut ainsi correspondre aussi bien à une exposition courte à forte concentration qu'à une exposition longue à faible concentration. Les niveaux d'intervention par rapport à la montée atmosphérique ont été choisis au regard de leurs correspondances avec la limite annuelle d'incorporation (LAI) et les niveaux opérationnels secondaires: niveau d'enregistrement (NE) et niveau d'investigation (NI). À chaque niveau le choix de l'examen le plus pertinent est guidé par les modèles biocinétiques (ICRP, 1989) et les limites de détection des examens de laboratoire (Tab. I). 


\section{TABLEAU II}

Conduite à tenir dans les premières heures suivant une contamination par inhalation. Procedures in case of exposure by inhalation during the first hours following the incident.

\begin{tabular}{|c|c|c|c|c|}
\hline serits a agrim & 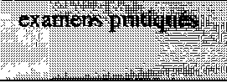 & 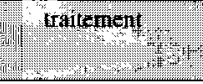 & 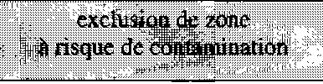 & 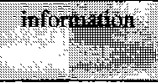 \\
\hline $\begin{array}{l}\text { LDCA } \cdot h<10 \\
\text { PN négatif }\end{array}$ & néant* & néant:* & néant* & néant* \\
\hline $\begin{array}{c}10<\mathrm{LDCA} h<60 \\
\text { PN négatif }\end{array}$ & $\begin{array}{l}\text { selles de } 48 \mathrm{~h} \\
\text { inítiales }\end{array}$ & nêtant* & aerant* & néant \\
\hline $\begin{array}{c}\text { LDCA } \cdot h>60 \\
\text { PN négatif }\end{array}$ & $\begin{array}{l}\text { selles de } 48 \mathrm{~h} \\
\text { urines de } 24 \mathrm{~h} \\
\text { anthroporadiamétrie }\end{array}$ & $\begin{array}{c}\text { DTPA spinhaler } \\
\text { puis } \\
\text { DTPA } \\
\text { 1/2 ampoule IVD } \\
\text { si } \\
\text { anthroporadiamét } \\
\text { rie significative }\end{array}$ & $\begin{array}{l}\text { Oui } \\
\text { si l'anthroporadiamétrie est non } \\
\text { significative, l'exclusion est } \\
\text { levée le jour où les prélèvements } \\
\text { sont rendus au laboratoire } \\
\text { si l'anthroporadiamétrie est } \\
\text { significative, la décision est } \\
\text { revue aux premiers résultats des } \\
\text { prélèvements }\end{array}$ & $\begin{array}{l}\text { le médecin } \\
\text { d'astreinte est } \\
\text { prévenu }\end{array}$ \\
\hline PN positif & $\begin{array}{l}\text { selles de } 48 \mathrm{~h} \\
\therefore \text { urines de } 24 \mathrm{~h} \\
\text { (trois jours } \\
\text { cortisecutifs) }\end{array}$ & $\begin{array}{c}\text { DTPA } \\
1 / 2 \text { amposile IVD }\end{array}$ & $\begin{array}{l}\text { Oui } \\
\text { si l'anthroporadiamétrie est non } \\
\text { significative, lexclusion est } \\
\text { levêe le jour où les prélevenentents } \\
\text { sont rendus au laboratoire }\end{array}$ & $\begin{array}{l}\text { le mécecin } \\
d^{3} \text { astreinte est } \\
\text { potécvenu }\end{array}$ \\
\hline : & anthroporadiamétrie & & 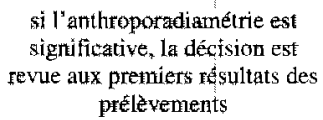 & \\
\hline
\end{tabular}

* Sauf circonstance particulière analysée par l'infinmier.

\section{Conduite à tenir en fonction des niveaux opérationnels (Tab. II)}

- $60 \mathrm{LDCA} \cdot \mathrm{h}$ est le niveau qui peut conduire à une exposition de $1 / 30^{\mathrm{e}}$ ( $60 \mathrm{~h} / 2000 \mathrm{~h}$ de travail) des limites annuelles (NE : niveau d'enregistrement). Pour cette raison, cette valeur a été choisie comme limite opérationnelle pour la décision d'une prescription d'examens complémentaires même en cas de PN « négatif ». À partir de ce niveau, une incorporation significative du point de vue réglementaire est à craindre, il est prescrit un traitement décorporant par acide diéthylène triamine penta-acétique (DTPA), des examens radiotoxicologiques, une exclusion de zone à risque de contamination ;

- entre 10 et $60 \mathrm{LDCA} \cdot \mathrm{h}$, seule l'analyse de selles présente une sensibilité suffisante. Cette analyse bien que prescrite dans une situation d'exposition a priori inférieure au NE ménage une marge de sécurité et la possibilité de prescrire des examens de contrôle en cas de positivité significative ; 
- au-dessous de 10 LDCA·h, aucun examen n'est prévu ;

- enfin tout PN positif déclenche systématiquement l'ensemble des examens, le traitement par une demi-ampoule soit 0,5 gramme de DTPA-intra-veineux direct (IVD) et une exclusion temporaire de zone à risque de contamination.

Rappelons que cette prescription s'appuie sur les principes suivants :

- l'excrétion fécale est le témoin le plus sensible d'une inhalation mais est souvent majorée dans les premières 48 heures par un passage digestif direct notamment de grosses particules (supérieures à 10 microns). Un contrôle après 10 jours permet de s'affranchir de ce mécanisme ;

- un résultat urinaire supérieur à la limite de détection signe l'absorption et le passage dans le compartiment systémique ;

- l'anthroporadiamétrie, une fois éliminée la contamination externe, apporte une information solide mais sa limite de détection pour les émetteurs alpha est faible.

L'application de ce protocole est régulée par l'analyse initiale des circonstances de l'incident faite par l'infirmier telles que la prise en compte d'informations aggravantes (contamination du visage, contamination cutanée avec solution acide...), ou d'imprécision sur le déroulement des évènements. C'est le sens de l'astérisque rajouté à «néant» dans le tableau II, qui prévoit la possibilité de prescription d'examens supplêmentaires.

Enfin, le port du masque lors de l'incident est pris en compte, l'infirmier s'assure qu'il était correctement porté et applique les mêmes consignes en divisant la LDCA-h d'un facteur 100 (facteur d'efficacité très inférieur à la réalité lorsque le masque est correctement porté).

En première urgence, l'infïrmier applique les protocoles ci-dessous.

En cas de positivité de l'anthroporadiamétrie initiale (limite de détection: $10 \mathrm{~Bq}$ en américium-24l fixée par notre laboratoire pour la composition isotopique du MOX), une incorporation d'au moins $25 \%$ de la LAI (Tab. I) peut être suspectée. Ce niveau ne pourra être validé que par les résultats des examens complémentaires radiotoxicologiques. Dans ce cas (Tab. III) :

- le traitement par DTPA IVD est poursuivi ;

- l'exclusion de zone est maintenue au moins jusqu'aux premiers résultats des examens radiotoxicologiques.

D'autre part l'anthroporadiamétrie sera répétée les jours suivants permettant de faire la part entre une inhalation et une contamination externe. Dans ce dernier cas, l'examen va rapidement devenir inférieur aux limites de détection (LD) (Tab. III). 


\section{TABLEAL III}

Conduite à tenir devant une contamination par inhalation dans les jours qui suivent l'incident. Procedures in case of exposure by inhalation during the days following the incident.

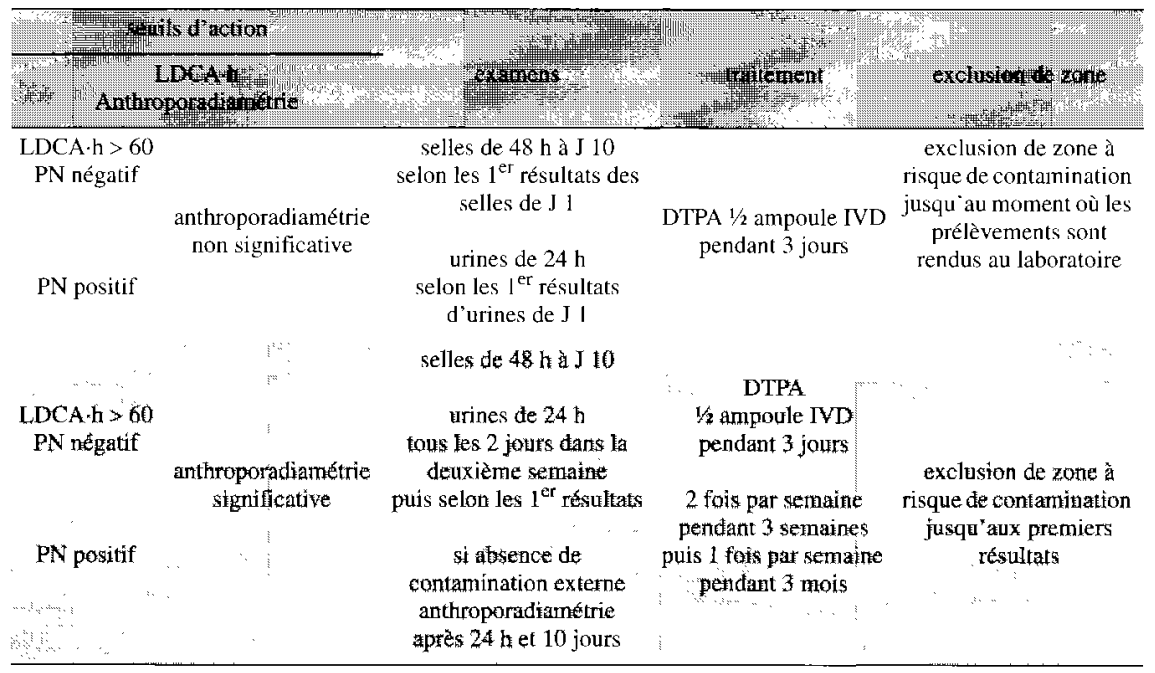

Le nombre et le type d'examens à prescrire initialement doivent prendre en compte l'imprécision de la connaissance des paramètres physico-chimiques et des conditions physiologiques des impliqués, et permettrent d'avoir suffisamment de résultats en vue de l'estimation de l'incorporation.

\subsubsection{Exposition par mécanisme transcutané ou percutané}

L'incorporation est évaluée par :

- les mesures au poste de travail sur l'objet contondant effectuées par le SPR ;

- les mesures sur la plaie par sonde X pratiquées au Laboratoire d'analyses médicales.

Si l'incorporation est d'emblée suspectée (plaie en boite à gants, sur objet contaminé, contamination en milieu acide ou solvant, peau abîmée) une injection de DTPA.IVD est effectuée dans les plus courts délais. Dans les autres cas la décision de traitement dépend du résultat de la sonde $X$.

La prescription d'analyse d'urines est systématiquement associée au traitement par DTPA.

L'exclusion de zone à risque de contamination est décidée au moins jusqu'à la cicatrisation de la plaie puis poursuivie en fonction des estimations dosimétriques à partir des premiers résultats radiotoxicologiques. 
TABLEAU IV

Conduite à tenir devant une contamination par plaie dans les premières heures de l'incident. Procedures in case of contamination due to a wound during the first hours following the incident.

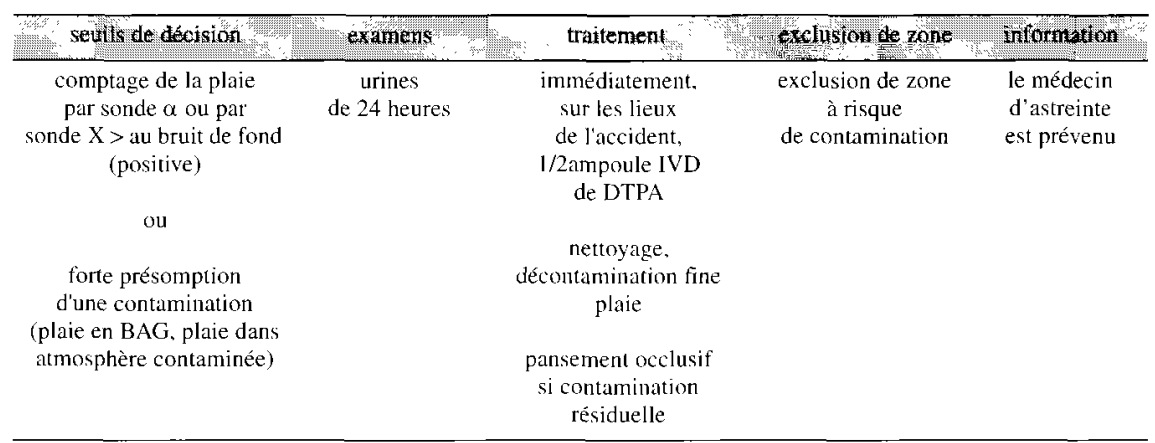

L'excision chirurgicale de la plaie contaminée n'entre pas dans les protocoles, cette décision est prise secondairement par le médecin. Cette indication est guidée par le risque de diffusion secondaire de la contamination à partir de fraction fixée au niveau de la plaie (Piechowski et al., 1992).

En cas de plaie grave, l'urgence médico-chirurgicale deviendrait prioritaire.

\subsection{Mise en place pratique et retour d'expérience}

La mise en place de ces protocoles n'a pu se faire qu'après un travail préalable de liaison avec les acteurs impliqués et de formation du personnel :

- la communication rapide entre le radioprotectionniste et l'infirmier de permanence, au début de l'incident a été grandement améliorée par l'élaboration conjointe d'une fiche d'information rapide (FIR), transmise par fax et recueillant toutes les informations nécessaires à la gestion de l'incident ;

- les protocoles ont été expliqués aux infïmiers. Une démarche d'information mutuelle par compagnonnage, leur en a permis l'appropriation et les a aidés à répondre aux salariés ;

- nous nous sommes impliqués dans la formation des salariés au risque radiologique, en présentant notre démarche de gestion des incidents. Nous avons privilégié une démarche interactive sollicitant l'expression des salariés et orientant les informations vers leurs réelles préoccupations ;

- les motifs de retrait de poste et de délais de réalisation des examens pour obtenir des résultats permettant l'évaluation de la dosimétrie interne ont été expliqués à la hiérarchie. Des procédures de liaisons pour l'informer rapidement d'un incident et des suites données ont été instaurées ; 
- nos procédures, dés leur élaboration, ont fait l'objet de présentations et de débats en CHSCT.

Rapidement, ces procédures de gestion d'incidents radiologiques ont répondu à nos objectifs initiaux :

- les infirmiers ont rencontré de moins en moins de difficulté pour expliquer et faire accepter les décisions médicales dans une phase souvent anxiogène pour le salarié ;

- les médecins du travail ont harmonisé leurs pratiques, surmontant ainsi un individualisme propre à la profession ;

- les agents de radioprotection ont pu communiquer rapidement les informations et ont progressivement enrichi la fiche d'information rapide ;

- les salariés ont eu l'occasion au cours des formations de s'exprimer sur leurs craintes et d'acquérir des points de repères sur les niveaux de dose et les risques pour leur santé ;

- les incidents de contamination et leur suivi médical sont régulièrement présentés au CHSCT ;

- les responsables ont compris nos raisonnements et les actions en découlant.

Ces protocoles, en resserrant nos indications, ont limité les contraintes des examens et des exclusions de risque de contamination.

\section{4. Évaluation et validation}

\subsection{Analyse des résultats en fonction des critères du protocole}

Notre objectif de départ pour la construction des protocoles était une évaluation initiale de l'incident, selon certains paramètres, avec la garantie de ne pas ignorer une incorporation significative ; tout en évitant une prescription systématique de tous les examens.

Les données concernant chaque incident sont enregistrées systématiquement dans le registre du bloc de décontamination. Leur exploitation informatique sur 8 années d'exercice nous permet de faire le retour d'expérience sur notre pratique et son adéquation par rapport à nos objectifs initiaux.

1529 incidents d'exposition ont été traités entre 1995 et 2003 parmi lesquels 1351 suspicions d'inhalation ( $88 \%$ ) et 173 suspicions de contamination par plaie $(11 \%)$. 
TABLEAU V

Répartition inhalations/plaies et doses.

Distribution inhalation/wounds and doses.

\begin{tabular}{ccc}
\hline & nombre de cas & nombre de doses retentes \\
\hline inhalations & 1351 & $18(1,3 \%)$ \\
plaies & 173 & $3(1,7 \%)$ \\
mixtes & 5 & $2(40 \%)$ \\
total & $\mathbf{1 5 2 9}$ & $\mathbf{2 3}(\mathbf{1 , 5} \%)$ \\
\hline
\end{tabular}

Les doses sont retenues lorsque l'incorporation estimée dépasse le 1/30 de la LAI (NE) et les calculs de dose sur la période retenue ont été faits en se référant à la surveillance dite spéciale telle que définie dans les modèles de la publication 54 de la CIPR (ICRP, 1989), à l'aide des rapports CEA (Piechowski et Menoux, 1984, 1985 ; Piechowski et al., 1992).

\subsection{Inhalations}

Les incorporations retenues sont comprises entre $4 \%$ et $100 \%$ de la LAI.

\subsubsection{Situations avec $P N$ négatif et $L D C A \cdot h<10$}

Il apparaît que la majorité des incidents pris en charge se situent au-dessous de nos critères de prescription d'examens et de traitement. Cependant, parmi eux, 128 ont fait l'objet de prescription (13\%) et 29 ont été traités (3\%) :

- dans la phase de mise en place, et pendant une campagne de trois mois avec prescription systématique sur tout incident (après débat en CHSCT) ;

- lors de situations jugées douteuses et prévues par l'astérisque du tableau.

Aucune dose n'a été retenue ce qui nous conforte dans l'abstention de prescription et de thérapeutique au-dessous de ces critères.

\subsubsection{Situations avec PN négatif et $10<L D C A \cdot h<60$}

Dans ces situations intermédiaires qui représentent 177 dossiers, soit $18 \%$ de nos cas, les investigations menées dans 134 cas ( $76 \%$ ), le plus souvent des selles, n'ont pas conduit à retenir de dose supérieure au NE.

Ces résultats pourraient remettre en question l'intérêt de poursuivre la prescription des selles dans ces circonstances. Nous conservons cette marge de sécurité prévue lors de l'élaboration des protocoles ( $c f$. Sect. 3.2.1) pour les raisons suivantes :

- nous observons fréquemment des positivités qui bien que se situant très endessous du niveau d'enregistrement permettent d'objectiver l'incorporation ; 


\section{TABLEAU VI}

Répartition des inhalations en fonction des critères du protocole et des doses. Distribution of inhalation according to the criteria of the protocol and the doses.

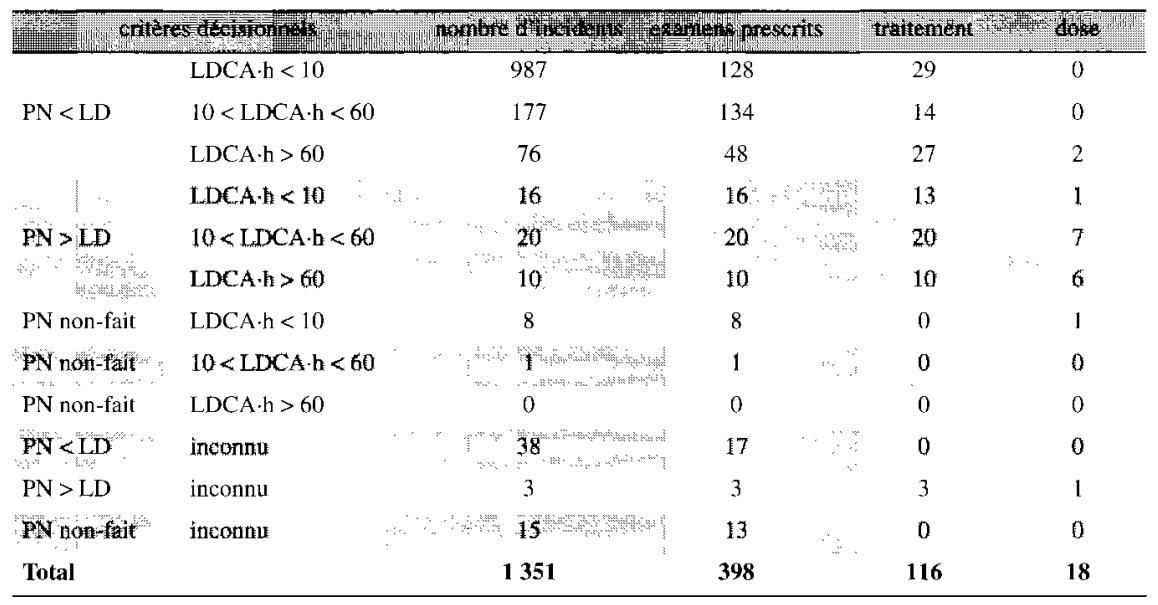

\section{TABLEAU VII}

Répartition des doses retenues en fonction des critères du protocole. Distribution of the doses according to the criteria of the protocol.

$\begin{array}{ll}\text { avec les } 2 \text { critères authentiftés } & 13 \\ \text { avec } 1 \text { des } 2 \text { critères } & 4 \\ \text { sans aucun critère } & 1\end{array}$

- l'absence de conséquence dosimétrique nous conforte dans l'abstention thérapeutique ;

- il est intéressant de pouvoir confronter les circonstances de l'évènement recueillies au poste de travail avec le résultat des investigations médicales (cf. Sect. 4.4.1).

\subsubsection{Autres situations}

Dans les circonstances, avec au moins un des deux critères positifs (cf. Sect. 3.2.1), le protocole est le plus souvent appliqué. L'écart constaté aux seconde et troisième lignes du tableau VI peut s'expliquer par quelques oublis lors de la mise en route du protocole. Ces cas surtout fréquents la première année illustrent la difficulté d'appropriation d'un protocole écrit.

Sur 18 doses retenues $(c f$. Tab. VII) :

- 13 le sont avec les deux critères positifs ; 


\section{TABLEAU VIII}

Corrélation entre doses et résultats de l'anthroporadiamétrie. Correlation between doses and results of spectrometry in vivo.

\begin{tabular}{|c|c|c|}
\hline 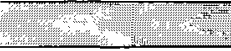 & anthropordiamétrie $>L D$ & 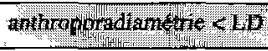 \\
\hline dose retenue ( $>$ NE) & 6 & 12 \\
\hline pas de dose ( $<\mathrm{NE}$ ) & 7 & 115 \\
\hline
\end{tabular}

- 4 avec un des deux critères ;

- 1 avec PN non-fait (enquête a posteriori) et LDCA-h $<10$.

Dans les 17 premiers cas, le traitement initial avait été instauré d'emblée.

Cette analyse valide nos choix décisionnels et l'importance de la prise en compte conjointe des deux indicateurs. En cas d'absence ou de difficulté d'interprétation de l'un d'eux, il convient de rester prudent. Dans ces cas, l'analyse des circonstances détaillées de l'incident et la connaissance des postes de travail guident alors le prescripteur.

\subsubsection{Intérêt de l'anthroporadiamétrie}

Au total, nous avons réalisé 140 examens ( $c f$. Tab. VIII).

L'anthroporadiamétrie est un examen pris en compte à un second niveau dans notre protocole. En effet :

- sa sensibilité est mauvaise dans la détection des actinides ;

- le délai de réalisation est d'environ une heure hors heure ouvrable, et ne doit pas retarder la mise en route du traitement ;

- enfin, la fréquence des contaminations externes associées rend son interprétation délicate.

Cependant, cet examen est intéressant dans les circonstances où l'on craint une incorporation significative (PN positif et/ou LDCA $h>60$ ) car lorsque la mesure est au-dessous des seuils de détection (cas le plus fréquent, $c f$. Tab. I), il permet de situer rapidement le niveau d'incorporation comme inférieur à la limite réglementaire et de fournir rapidement un élément rassurant.

\subsection{Les plaies}

Toute plaie même cliniquement insignifiante peut entraîner un passage sanguin important (Piechowski et al., 2003). La rapidité de fixation aux organes cibles depuis le compartiment systémique justifie l'urgence thérapeutique (Piechowski et al., 1992). Le DTPA sera effectué au plus vite par l'infirmier sur les lieux du 
travail devant toute présomption de contamination : plaie en $\mathrm{BAG}$, en atmosphère contaminée ou sur matériel contaminé...

Dans un second temps, la mesure de la plaie par sonde $X$ sera systématiquement réalisée au LABM. Sur 173 expositions par plaie 8 fois la détection à la sonde $\mathrm{X}$ a été positive, 3 doses ont été retenues parmi ces 8 cas. Dans les cas avec sonde $X$ négative, des examens ont été prescrits 94 fois et aucune dose n'a été retenue. Cependant, la fréquence de positivités minimes, inférieures au niveau d'enregistrement dérivé (NED) dans les urines traduit des passages systémiques. La sonde $\mathrm{X}$ ne peut donc être le seul critère de décision.

\subsection{Discussion}

\subsubsection{Corrélation entre le niveau de concentration atmosphérique et l'incorporation retenue}

Dans tous les cas où ont été prescrits des examens radiotoxicologiques (398) l'incorporation a été estimée. Cela nous permet de corréler ce résultat avec celui extrapolé à partir du niveau de la concentration atmosphérique.

Nous observons une mauvaise corrélation entre la concentration atmosphérique et l'incorporation estimée par les mesures individuelles :

- 86 situations avec LDCA.h $>60$ : seules 8 doses ont été retenues ;

- sur les 18 doses retenues au total, le niveau de l'incorporation estimée est souvent très supérieur à celui que laissait prévoir celui de la montée atmosphérique.

Cette mauvaise corrélation est rapportée dans le paragraphe 4.3 de la publication 78 de la CIPR (ICRP, 1997) avec une sous estimation atteignant un facteur 10 retrouvé dans nos observations.

L'origine de ces écarts est probablement multifactorielle, notamment :

- position des détecteurs atmosphériques par rapport au salarié ;

- méconnaissance de tous les paramètres physico-chimiques de la contamination avec utilisation des paramètres par défaut de la CIPR.

Au total, la surveillance en continu des atmosphères de travail telle qu'elle est faite à Cadarache est un outil performant de radioprotection indispensable au médecin mais qui ne peut suppléer aux mesures sur l'individu pour estimer une incorporation. Il est nécessaire de conserver une marge de manouvre dans leur prise en compte. 


\subsubsection{Intérêt du suivi systématique}

Des positivités sont parfois découvertes par la surveillance systématique périodique dont bénéficient tous les agents ayant un risque d'exposition significatif. Les incorporations retenues dans ces circonstances sont rares (une par an environ) et inférieures au quart de la LAI. Les investigations complémentaires menées dans ces cas n'ont jamais permis de retrouver une corrélation avec un évènement identifié.

Cette surveillance conforte nos protocoles « incidents » et apporte un niveau de contrôle supplémentaire d'éventuelles expositions infraliminaires.

\subsubsection{Aspects thérapeutiques}

Le DTPA n'ayant pas fait l'objet d'une autorisation de mise sur le marché (AMM), nous nous appuyons sur les recommandations des organismes de tutelle (OPRI, 1997) ou d'expertise (CEC, DOE et IPSN, 1995 ; IAEA, 1978, 1996 ; IAEA, OMS et BIT, 1981, 1988) pour nous guider dans nos prescriptions. Dans les différentes publications disponibles, les indications du traitement varient de « devant toute suspicion de contamination » (OPRI, 1997) ou a priori (OPRI, 2001, circulaire DHOS/HFD/DGSNR 277 du 2 mai 2002) à «contamination susceptible d'avoir dépassé une LAI » (CEC, DOE et IPSN, 1995). La notion d'urgence thérapeutique sans attendre d'avoir la preuve formelle de cette contamination est par contre unanimement retenue.

Dans notre contexte, caractérisé par la fréquence des évènements à risque d'inhalation, nous avons limité l'indication de traitement aux situations susceptibles d'entraîner une incorporation supérieure au niveau d'enregistrement. L'administration du DTPA par l'infirmier en application du protocole permet la maîtrise de ces indications.

Pour les inhalations, la prescription du traitement est associée à celle des examens à visée dosimétrique. En appliquant notre protocole, 81,4\% des personnes impliquées n'ont pas été traitées. Aucune d'entre elles n'a eu de dose retenue.

Pour les plaies, compte tenu de la difficulté à évaluer initialement le risque d'incorporation, la mise en route du traitement est plus systématique. Le traitement est fait selon l'évaluation de la situation par l'infirmier ( $c f$. Tab. IV), ce qui représente $50 \%$ des cas.

La posologie utilisée à Cadarache est de $0,5 \mathrm{~g}$ par injection selon les recommandations d'un groupe d'experts du CEA et de l'Institut Curie (OMS, Institut Curie et CEA, 1984). 
Nous n'avons pas constaté dans notre pratique d'effets iatrogènes du DTPA.

Le traitement est renouvelé en fonction des critères d'évaluation initiaux jusqu'aux premiers résultats radiotoxicologiques urinaires.

L'efficacité du traitement s'évalue par comparaison des excrétions avant et après injection. Le gain dosimétrique s'évalue par l'évolution de l'excrétion au cours du temps par rapport aux résultats initiaux et guide la poursuite ou l'arrêt du traitement. Dans la majorité des cas, le traitement chélateur n'est pas poursuivi car l'élimination urinaire devient très vite inférieure aux limites de détection. Dans les cas plus importants où l'élimination urinaire persiste en diminuant progressivement, le traitement est poursuivi en suivant le tarissement du compartiment systémique dont témoigne l'évolution des résultats urinaires (Piechowski et al., 2003). L'arrêt du traitement sera argumenté par l'épuisement apparent de l'effet du DTPA jugé sur la comparaison des résultats urinaires avant et après injection et sur l'évolution de ceux-ci par rapport aux résultats initiaux.

\section{Conclusion}

La gestion médicale des incidents de contamination par les transuraniens est sous tendue par deux objectifs principaux : la mise en route précoce d'un traitement et l'évaluation de la dose dans les situations d'incorporation significative. On entend par-là et de façon consensuelle toute incorporation d'un niveau supérieur à $1 / 30^{\mathrm{e}}$ de la LAI (NE) (ICRP, 1989 ; Piechowski et Menoux, 1984 ; CEA et COGEMA, 2001 ; CEC, DOE et IPSN, 1995).

Le problème qui se pose au médecin du travail de l'INB est d'avoir à prendre en urgence des décisions à la fois thérapeutiques et diagnostiques permettant une estimation de dose avant même d'avoir une évaluation directe possible du niveau d'incorporation. Nos protocoles permettent de guider cette décision par une prise en compte d'éléments d'orientation indirects mais immédiats.

Cette démarche médicale fondée sur les réflexions des groupes de travail des médecins et biologistes du groupe CEA, depuis plus de vingt ans se trouve validée par ce retour d'expérience sur la gestion des situations incidentelles. Elle est indissociable de la surveillance radiotoxicologique de routine et du dispositif de radioprotection au poste de travail. Elle n'est pas remise en cause par la nouvelle réglementation mais demande une adaptation de nos protocoles avec de nouvelles valeurs opérationnelles. Ce travail est en cours.

\section{RÉFÉRENCES}

CEA, COGEMA (2001) Guide de surveillance radiotoxicologique des travailleurs exposés au risque d'inhalation d'actinides. Groupe technique des SMT et LABM CEA et COGEMA, 2001. 


\section{PROTOCOLES DE PRISE EN CHARGE DES INCIDENTS}

CEC, DOE et IPSN (1995) Guidebook for the treatement of accidental internal ranucleide contamiantion of workers, Radiation Protection Dosimetry (G.B. Gerber, R.G. Thomas, Eds.). Nuclear Technology Publishing, USA. Traduction française : Traitement de la contamination interne accidentelle des travailleurs, IPSN, 1995.

Cérat D. (1994) EDGAR, Retour d'expérience, Rayonnements Ionisants (4), 11-33.

Code du travail :

- arrêté du 28 août 1991 recommandations aux médecins du travail assurant la surveillance médicale des travailleurs exposés aux rayonnements ionisants ;

- circulaire DHOS/HFD/DGSNR 277 du 2 mai 2002 relative à l'organisation des soins médicaux en cas d'accident nucléaire ou radiologique ;

- décret $n^{\circ}$ 66-450 du 20 juin 1966 relatif aux principes généraux de protection contre les rayonnements ionisants ;

- décret $n^{\circ} 75-306$ du 28 avril 1975 relatif à la protection des travailleurs contre les dangers des rayonnements ionisants dans les INB ;

- décret $n^{\circ} 86-1103$ du 02 octobre 1986 , relatif à la protection des travailleurs contre les dangers des rayonnements ionisants ;

- $\quad$ décret $n^{\circ} 88-662$ du 06 mai 1988 modifiant le décret n ${ }^{\circ}$ 75-306 du 28 avril 1975 ;

- décret no 91-963 du 19 septembre 1991 ;

- décret $n^{\circ} 2003-296$ du 31 mars 2003 relatif à la protection des travailleurs contre les dangers des rayonnements ionisants.

IAEA (1978) Treatement of incorporated trasuranium element, Technical Report Series No. 184, Vienna, Austria.

IAEA (1996) Assesment and treatment of external and internal radionuclide contamination, TECDOC-869, Vienna, Austria.

IAEA, OMS et BIT (1981) Safety Series No. 47, Vienna, Austria.

IAEA, OMS et BIT (1988) Safety Series No. 88, Vienna, Austria.

ICRP Publication 54 (1989) Individual Monitoring for Intakes of Radionuclides by Workers: Design and Interpretation, Ann. ICPR 19(1-3).

ICRP Publication 78 (1997) Individual Monitoring for Internal Exposure of Workers: Replacement of ICRP Publication 54, Ann. ICRP 27(3-4).

OMS, Institut Curie et CEA (1984) Collection 84, 03, Accident radiologique, conduite à tenir en cas de surexposition, Fontenay-aux-Roses.

OPRI (1997) Conseil scientifique de l'Office de protection contre les rayonnements ionisants, Intervention médicale en cas d'accident radiologique.

OPRI (2001) Conseil scientifique de l'Office de protection contre les rayonnements ionisants, Intervention médicale en cas d'accident radiologique.

Piechowski J., Menoux B. (1984) Rétention et excrétion des radionucléides après incorporation par inhalation chez l'homme adulte, rapport CEA-R-5266-S, CE Saclay, Gif-sur-Yvette.

Piechowski J., Menoux B. (1985) Protocole de surveillance individuelle pour les contaminations par inhalation de radionucléides en milieu professionnel, rapport CEA-R-5305, CE Saclay, Gif-surYvette.

Piechowski J., Menoux B., Chaptinel Y. (1992) Évaluation de l'exposition systémique résultant d'une blessure contaminée par des produits radioactifs, rapport CEA-R-5583, CE Saclay, Gif-surYvette.

Piechowski J., Menoux B., Miele A., Grappin L., Guillermin A.-M., Fottorino R., Ruffin M. (2003) Implication du médecin du travail et le l'expert dans la gestion et la dosimétrie d'un incident de contamination : exemple d'une plaie contaminée par le plutonium, Radioprotection 38(1), 29-50. 